\title{
Correspondence
}

\section{Anesthesia research productivity in Canadian universities: a full picture?} (I)

To the Editor:

We are grateful that Tsui et al. ${ }^{1}$ have presented serious concerns about the status of anesthesia research in Canada in the March 2006 issue of the Journal. We believe that a resident's decision to pursue research fellowship training and a research career depends on many factors, including the desire for an academic career (which probably depends on exposure and role modelling during residency), lifestyle, current debt, future income expectations, institutional culture, and research infrastructure and support. With the current anesthesia shortage, it is difficult to convince an outstanding resident to take on additional years of research training if the institution or department derogates the value of research and researchers. This is particularly problematic in institutions where a practice plan or an alternative funding program is not available to protect academic time.

Although Tsui et al. provide interesting information, we are concerned about the inaccurate portrayal of "publication rates" for various universities, which appears to paint a biased picture of research prowess of the bigger institutions. Tsui and colleagues suggest that limitations related to collaborative publication across departments "would be common to all Canadian universities in our search", but we believe that is incorrect. In their report, Dalhousie was credited with only 13 papers in five years. However, a quick search in Medline revealed that just three of the several researchers in our institution had a total of 53 publications during that period of time. So the reported number grossly underestimates the productivity of our institution, hardly a "slight under representation". Perhaps, this is, in part, due to the search criteria used in their study (i.e., searching for "anesthesia" or its variants in the "address" field on Medline). It may be more common for researchers in small departments to collaborate with colleagues in other disciplines simply because there is less range of expertise within their own department, or to supervise research students from other disciplines. For example, many of our faculty members have cross appointments in other departments (e.g., psychology, pharmacology, surgery, internal medicine, critical care, and pain research), so corresponding addresses may not have included the term "anesthesia". If Tsui et al. have reported an equivalent four- or fivefold underestimate of publication numbers for all the universities in their study, then there is little support for their conclusion that anesthesia research has been static over the time period, nor for their interpretation of publication types. If their inaccuracy is disproportionately focused on smaller departments, as we believe, then there is a clearly biased portrayal of research productivity that may be directly harmful, particularly to faculty recruitment efforts. In any case, reporting a "lack" of research productivity using an inaccurate measure and a narrow search strategy is potentially damaging to our specialty.

Notwithstanding these issues, we would echo the investigators' concerns about the future of Canadian anesthesia research. A strategic plan to address funding issues, research infrastructure, and institutional culture is certainly needed.

\section{G. Allen Finley MD FRCPC FAAP}

Orlando Hung MD FRCPC

Thomas J. Coonan MD FRCPC

Richard Hall MD FRCPC

Dalhousie University, Halifax, Canada

E-mail: allen.finley@dal.ca

Accepted for publication May 2, 2006.

\section{Reference}

I Tsui BC, Li LX, Ma V, Wagner AM, Finucane BT. Declining randomized clinical trials from Canadian anesthesia departments? Can J Anesth 2006; 53: 22635 .

\section{Anesthesia research productivity in Canadian universities: a full picture?} (II)

To the Editor:

Tsui et al. ${ }^{1}$ reported on the number of published articles from the Canadian departments of anesthe- 